\title{
NOx Emissions Cost Assessment Due to Rapid Mass Transport Development Plan (Tram) in Surabaya
}

\author{
Mochammad Choirul Rizal $^{1 *}$, Hera Widyastuti ${ }^{2}$ \\ ${ }^{1}$ Surabaya Shipbuilding State Polytechnic \\ ${ }^{2}$ Sepuluh Nopember Institute of Technology Surabaya \\ *Corresponding author: mochammadchoirulrizal@yahoo.com
}

\begin{abstract}
One program Surabaya City Government to repair the transport sector is build a mass transit rapid that one of them is a tram. The rapid development of mass transit is also expected to support the improvement of air quality in the city of Surabaya due to the effects of vehicle emissions. On of harmful vehicle emissions are Nox. One danger is the Nox can cause lung inflammation and paralysis. On the other hand, rapid mass transit development plan in Surabaya planned to use a portion of the road as the tram line. This will certainly reduce the capacity of roads. Therefore, a comparison between a reduction in the number of private vehicle users with the capacity reduction of the cost of air pollution in this case is the cost of Nox emissions into the topic of this research. The location of this research is Basuki Rahmat Street and Urip Sumoharjo Street which is one of the roads that traversed the tram line which is often congested. The method of calculating the amount of Nox based on the vehicle speed on the exixting condition and the condition of the plan. Based on calculations, the level of Nox reduction in the time before the tram operates when compared to when the tram operates on Urip Sumoharjo Street amounted 7.38\%. While the level of Nox reduction in the time before the tram operates when compared to when the tram operates on Basuki Rahmat Street amounted to $11.81 \%$. Then from the calculation of the cost air pollution, decrease the cost of air pollution due to Nox in Urip Sumoharjo Street is 9,185,321.87 rupiahs/year, while the decline in the cost of air pollution due to Nox in Basuki Rahmat Street is 14,664,554.40 rupiahs/year.
\end{abstract}

Keywords—emission, nitrogen oxide, mass rapid transit, modal split.

\section{INTRODUCTION}

Cong ongestion is one of the problems in the field of transportation that often occurs in several major cities, one of which is in Surabaya. The derivation of the congestion problem is certainly a matter of air quality. Discussion on air quality issues, can not be separated from the discussion of exhaust emissions. One of the largest sources of exhaust emissions is the transportation sector. The increasing number of vehicles from year to year, causing congestion on some roads, resulting in decreasing air quality in a region.

Air pollution is the entry or inclusion of living things, substances, energies and or changes of the air order (composition) by human activities or by natural processes so that air quality becomes less or can no longer function according to its designation (Kusuma, 2013) ${ }^{[1]}$.

There are some exhaust emissions arising from highway traffic, one of which is a dangerous $\mathrm{NO}_{\mathrm{x}}$ gas. When the $\mathrm{NO}_{\mathrm{x}}$ gas reacts with water $\left(\mathrm{H}_{2} \mathrm{O}\right)$ it will produce nitric acid. The nature of this nitric acid can cause corrosion.

The congestion road in Surabaya City is the middle road section of the city linking the north-south corridor, some of which are Basuki Rahmat Street and Urip Sumoharjo Street (Figure 1). To reduce the congestion, one of the plans proposed by Surabaya City Government is to build fast mass transit in the form of tram. Then how will the $\mathrm{NO}_{\mathrm{x}}$ emissions change amount if the tramway development plan is carried out and if the tram construction plan is not carried out and what is the estimated cost of air pollution due to $\mathrm{NO}_{\mathrm{x}}$ emissions for both conditions? This will be the topic of study in this research, which is under the title of $\mathrm{NO}_{\mathrm{x}}$ Emissions Cost Assessment Due to Rapid Mass Transport Development Plan (Tram) In Surabaya.

\section{METHOD}

In this study, the approach used is theoretical approach. The method used to predict the amount of $\mathrm{NO}_{x}$ gas emissions is using the Pedoman Teknik Tata Cara Prediksi Polusi Udara Skala Mikro Akibat Lalu Lintas No. 017/T/BM/1999 ${ }^{[2]}$. The predicted number of $\mathrm{NO}_{\mathrm{x}}$ gas emissions is done for each condition, i.e. before the tram operates and at the time after the tram operates.

The data used are traffic volume data during peak hours, road geometric data, and modal split data.To calculate the amount of emission of nitrogen oxide $\left(\mathrm{NO}_{\mathrm{x}}\right)$ gas, the initial step is to normalize the volume of the vehicle to the passenger car unit. The way to normalize the volume of vehicles to units of passenger cars is to use the $\mathrm{NO}_{\mathrm{x}}$ multiplier factor table based on the type of vehicle (Table 1).

The next step after the normalization of vehicle volume is to calculate the emission rate. The emission rate is calculated by entering the vehicle density variables in the road segment under review using the equation $\mathrm{qNO}_{\mathrm{x}}=0.0005 \mathrm{~V} 2-0.0656 \mathrm{~V}+3.6586$, with $\mathrm{V}$ is the average vehicle speed $(\mathrm{km} / \mathrm{h})$.

Then for the percentage of modal split, using secondary data from the research ever conducted.

\section{RESULTS AND DISCUSSION}

Vehicle volume survey was conducted from 06.00 a.m. until 10.00 p.m. From the results of the survey volume of 
The Third International Conference on Civil Engineering Research (ICCER)

August $1^{\text {st }}-2^{\text {nd }} 2017$, Surabaya - Indonesia

Table 1. Multiplier Factor for $\mathrm{NO}_{\mathrm{x}}$ Emissions (Puslitbang, 1999)

\begin{tabular}{ccccc}
\hline \multirow{2}{*}{ Type of Vehicle } & \multicolumn{4}{c}{ Multiplier Factor For NO $\mathbf{X}_{\mathbf{x}}$ Emissions } \\
\cline { 2 - 5 } & Metropolitan & Big City & Medium City & Others \\
\hline Motor Cycle (MC) & 0.6 & 0.6 & 0.6 & 0.6 \\
\hline Light Vehicle (LV) & 1 & 0.81 & 0.84 & 0.76 \\
\hline Heavy Vehicle (HV) & 1.45 & 1.46 & 1.45 & 1.93 \\
\hline
\end{tabular}

Table 2. Existing Vehicle Volume Data at Peak Hours

\begin{tabular}{lcccccc}
\hline \multirow{2}{*}{ Location } & \multicolumn{3}{c}{$\begin{array}{c}\text { Direction 1 } \\
\text { (vehicle/second) }\end{array}$} & \multicolumn{3}{c}{$\begin{array}{c}\text { Direction 2 } \\
\text { (vehicle/second) }\end{array}$} \\
\cline { 2 - 7 } & MC & LV & HV & MC & LV & HV \\
\hline Jl. Urip Sumoharjo & & & & & & \\
(6/2 D) & & & & & & \\
\hline Morning & 2.4600 & 1.0547 & 0.0028 & 1.8097 & 0.5122 & 0.0022 \\
\hline Afternoon & 1.9147 & 1.1736 & 0.0044 & 1.3753 & 0.5572 & 0.0017 \\
\hline Evening & 1.3381 & 0.6553 & 0.0008 & 3.1161 & 0.9297 & 0.0008 \\
\hline Jl. Basuki Rahmat & & & & & & \\
(5/1 D) & & & & & & \\
\hline Morning & 4.1883 & 3.1386 & 0.0014 & - & - & - \\
\hline Afternoon & 3.9956 & 3.6392 & 0.0008 & - & - & - \\
\hline Evening & 4.7403 & 1.7181 & 0.0011 & - & - & - \\
\hline
\end{tabular}


The Third International Conference on Civil Engineering Research (ICCER)

August $1^{\text {st }}-2^{\text {nd }}$ 2017, Surabaya - Indonesia

Table 3. Vehicle Volume Data Condition Plan Before Tram Operates

\begin{tabular}{lcccccc}
\hline \multirow{2}{*}{ Location } & \multicolumn{3}{c}{$\begin{array}{c}\text { Direction 1 } \\
\text { (vehicle/second) }\end{array}$} & \multicolumn{3}{c}{$\begin{array}{c}\text { Direction 2 } \\
\text { (vehicle/second) }\end{array}$} \\
\cline { 2 - 7 } & MC & LV & HV & MC & LV & HV \\
\hline Jl. Urip Sumoharjo & & & & & & \\
(6/2 D) & & & & & & \\
\hline Morning & 3.0310 & 1.1851 & 0.0029 & 2.2298 & 0.5755 & 0.0024 \\
\hline Afternoon & 2.3591 & 1.3187 & 0.0047 & 1.6945 & 0.6261 & 0.0018 \\
\hline Evening & 1.6486 & 0.7363 & 0.0009 & 3.8394 & 1.0446 & 0.0009 \\
\hline Jl. Basuki Rahmat & & & & & & \\
(5/1 D) & & & & & & \\
\hline Morning & 5.1604 & 3.5265 & 0.0015 & - & - & - \\
\hline Afternoon & 4.9229 & 4.0890 & 0.0009 & - & - & - \\
\hline Evening & 5.8405 & 1.9304 & 0.0012 & - & - & - \\
\hline
\end{tabular}

Table 4. Vehicle Volume Data Condition Plan If Tram Operates

\begin{tabular}{lcccccc}
\hline \multirow{2}{*}{ Location } & \multicolumn{3}{c}{$\begin{array}{c}\text { Direction 1 } \\
\text { (vehicle/second) }\end{array}$} & \multicolumn{3}{c}{$\begin{array}{c}\text { Direction 2 } \\
\text { (vehicle/second) }\end{array}$} \\
\cline { 2 - 7 } & MC & LV & HV & MC & LV & HV \\
\hline $\begin{array}{l}\text { Jl. Urip Sumoharjo } \\
\text { (6/2 D) }\end{array}$ & & & & & & \\
\hline Morning & 2.2602 & 0.8837 & 0.0029 & 1.6627 & 0.4292 & 0.0024 \\
\hline Afternoon & 1.7592 & 0.9833 & 0.0047 & 1.2636 & 0.4669 & 0.0018 \\
\hline Evening & 1.2294 & 0.5490 & 0.0009 & 2.8630 & 0.7790 & 0.0009 \\
\hline Jl. Basuki Rahmat & & & & & & \\
(5/1 D) & & & & & & \\
\hline Morning & 3.8481 & 2.6297 & 0.0015 & - & - & - \\
\hline Afternoon & 3.6710 & 3.0491 & 0.0009 & - & - & - \\
\hline Evening & 4.3553 & 1.4395 & 0.0012 & - & - & - \\
\hline
\end{tabular}

Table 5. Conversion of Existing $\mathrm{NO}_{\mathrm{x}}$ Emission Factor

\begin{tabular}{lcccccc}
\hline \multirow{2}{*}{ Location } & \multicolumn{3}{c}{$\begin{array}{c}\text { Direction 1 } \\
\text { (units of passenger cars/second) }\end{array}$} & \multicolumn{2}{c}{ Direction 2 } \\
& (units of passenger cars/second) \\
\cline { 2 - 7 } & MC & LV & HV & MC & LV & HV \\
\hline Jl. Urip Sumoharjo & & & & & & \\
(6/2 D) & & & & & & \\
\hline Morning & 1.4760 & 1.0547 & 0.0040 & 1.0858 & 0.5122 & 0.0032 \\
\hline Afternoon & 1.1488 & 1.1736 & 0.0064 & 0.8252 & 0.5572 & 0.0024 \\
\hline Evening & 0.8028 & 0.6553 & 0.0012 & 1.8697 & 0.9297 & 0.0012 \\
\hline Jl. Basuki Rahmat & & & & & & \\
(5/1 D) & & & & & & \\
\hline Morning & 2.5130 & 3.1386 & 0.0020 & - & - & - \\
\hline Afternoon & 2.3973 & 3.6392 & 0.0012 & - & - & - \\
\hline Evening & 2.8442 & 1.7181 & 0.0016 & - & - & - \\
\hline
\end{tabular}

Table 6. Conversion of $\mathrm{NO}_{\mathrm{x}}$ Emission Factor Condition Plan Before Tram Operates

\begin{tabular}{lcccccc}
\hline \multirow{1}{*}{ Location } & \multicolumn{3}{c}{$\begin{array}{c}\text { Direction 1 } \\
\text { (units of passenger cars/second) }\end{array}$} & \multicolumn{3}{c}{ Direction 2 } \\
& (units of passenger cars/second) \\
\cline { 2 - 7 } & MC & LV & HV & MC & LV & HV \\
\hline $\begin{array}{l}\text { Jl. Urip Sumoharjo } \\
\text { (6/2 D) }\end{array}$ & & & & & & \\
\hline Morning & 1.8186 & 1.1851 & 0.0043 & 1.3379 & 0.5755 & 0.0034 \\
\hline Afternoon & 1.4155 & 1.3187 & 0.0068 & 1.0167 & 0.6261 & 0.0026 \\
\hline Evening & 0.9892 & 0.7363 & 0.0013 & 2.3036 & 1.0446 & 0.0013 \\
\hline Jl. Basuki Rahmat & & & & & & \\
(5/1 D) & & & & & & \\
\hline Morning & 3.0963 & 3.5265 & 0.0021 & - & - & - \\
\hline Afternoon & 2.9538 & 4.0890 & 0.0013 & - & - & - \\
\hline Evening & 3.5043 & 1.9304 & 0.0017 & - & - & - \\
\hline
\end{tabular}


The Third International Conference on Civil Engineering Research (ICCER)

August $1^{\text {st }}-2^{\text {nd }}$ 2017, Surabaya - Indonesia

Table 7. Conversion of $\mathrm{NO}_{x}$ Emission Factor Condition Plan If Tram Operates

\begin{tabular}{lcccccc}
\hline \multirow{2}{*}{ Location } & \multicolumn{3}{c}{$\begin{array}{c}\text { Direction 1 } \\
\text { (units of passenger cars/second) }\end{array}$} & \multicolumn{3}{c}{$\begin{array}{c}\text { Direction 2 } \\
\text { (units of passenger cars/second) }\end{array}$} \\
\cline { 2 - 7 } & $\mathbf{M C}$ & $\mathbf{L V}$ & $\mathbf{H V}$ & $\mathbf{M C}$ & $\mathbf{L V}$ & $\mathbf{H V}$ \\
\hline $\begin{array}{l}\text { Jl. Urip Sumoharjo } \\
\text { (6/2 D) }\end{array}$ & & & & & & \\
\hline Morning & 1.3561 & 0.8837 & 0.0043 & 0.9976 & 0.4292 & 0.0034 \\
\hline Afternoon & 1.0555 & 0.9833 & 0.0068 & 0.7581 & 0.4669 & 0.0026 \\
\hline Evening & 0.7376 & 0.5490 & 0.0013 & 1.7178 & 0.7790 & 0.0013 \\
\hline Jl. Basuki Rahmat & & & & & & \\
(5/1 D) & & & & & & \\
\hline Morning & 2.3089 & 2.6297 & 0.0021 & 2.3089 & 2.6297 & 0.0021 \\
\hline Afternoon & 2.2026 & 3.0491 & 0.0013 & 2.2026 & 3.0491 & 0.0013 \\
\hline Evening & 2.6132 & 1.4395 & 0.0017 & 2.6132 & 1.4395 & 0.0017 \\
\hline
\end{tabular}

Table 8. Vehicle Speed Calculation Results Existing Condition

\begin{tabular}{lcccrrrr}
\hline \multirow{1}{*}{ Location } & \multicolumn{3}{c}{$\begin{array}{c}\text { Direction 1 } \\
\text { (km/hour) }\end{array}$} & & & \multicolumn{3}{c}{$\begin{array}{c}\text { Direction 2 } \\
\text { (km/hour) }\end{array}$} \\
\cline { 2 - 8 } & & MC & LV & HV & MC & LV & HV \\
\hline J1. Urip Sumoharjo & & & & & & & \\
(6/2 D) & & & & & & & \\
\hline Morning & 9.26 & 3.16 & 1.32 & 13.22 & 4.77 & 2.05 \\
\hline Afternoon & 9.21 & 3.14 & 1.31 & 13.57 & 4.92 & 2.12 \\
\hline Evening & 12.77 & 4.58 & 1.96 & 9.33 & 3.19 & 1.33 \\
\hline J1. Basuki Rahmat & & & & & & \\
(5/1 D) & & & & & & \\
\hline Morning & 4.85 & 1.56 & 0.63 & - & - & - \\
\hline Afternoon & 4.30 & 1.36 & 0.55 & - & - & - \\
\hline Evening & 7.05 & 2.35 & 0.97 & - & - & - \\
\hline
\end{tabular}

Table 9. Vehicle Speed Calculation Results Condition Plan Before Tram Operates

\begin{tabular}{|c|c|c|c|c|c|c|}
\hline \multirow[t]{2}{*}{ Location } & \multicolumn{3}{|c|}{$\begin{array}{c}\text { Direction } 1 \\
(\mathbf{k m} / \mathbf{h o u r})\end{array}$} & \multicolumn{3}{|c|}{$\begin{array}{c}\text { Direction } 2 \\
\text { (km/hour) }\end{array}$} \\
\hline & MC & LV & HV & MC & LV & HV \\
\hline \multicolumn{7}{|c|}{$\begin{array}{l}\text { Jl. Urip Sumoharjo } \\
\text { (6/2 D) }\end{array}$} \\
\hline Morning & 8.31 & 2.80 & 1.16 & 12.15 & 4.32 & 1.84 \\
\hline Afternoon & 8.30 & 2.80 & 1.16 & 12.55 & 4.48 & 1.92 \\
\hline Evening & 11.78 & 4.17 & 1.77 & 8.33 & 2.80 & 1.16 \\
\hline \multicolumn{7}{|c|}{$\begin{array}{l}\text { Jl. Basuki Rahmat } \\
\text { (5/1 D) }\end{array}$} \\
\hline Morning & 4.16 & 1.32 & 0.53 & - & - & - \\
\hline Afternoon & 3.65 & 1.14 & 0.45 & - & - & - \\
\hline Evening & 6.20 & 2.04 & 0.83 & - & - & - \\
\hline
\end{tabular}

Table 10. Vehicle Speed Calculation Results Condition Plan If Tram Operates

\begin{tabular}{lcccccc}
\hline \multirow{2}{*}{ Location } & \multicolumn{3}{c}{$\begin{array}{c}\text { Direction 1 } \\
\text { (km/hour) }\end{array}$} & \multicolumn{3}{c}{$\begin{array}{c}\text { Direction 2 } \\
\text { (km/hour) }\end{array}$} \\
\cline { 2 - 7 } & MC & LV & HV & MC & LV & HV \\
\hline $\begin{array}{l}\text { Jl. Urip Sumoharjo } \\
\text { (6/2 D) }\end{array}$ & & & & & & \\
\hline Morning & 5.98 & 1.94 & 0.79 & 9.51 & 3.26 & 1.36 \\
\hline Afternoon & 5.97 & 1.94 & 0.79 & 9.89 & 3.41 & 1.43 \\
\hline Evening & 9.24 & 3.15 & 1.32 & 5.92 & 1.92 & 0.78 \\
\hline Jl. Basuki Rahmat & & & & & & \\
(5/1 D) & & & & & & \\
\hline Morning & 4.25 & 1.35 & 0.54 & - & - & - \\
\hline Afternoon & 3.73 & 1.17 & 0.47 & - & - & - \\
\hline Evening & 6.29 & 2.07 & 0.85 & - & - & - \\
\hline
\end{tabular}


The Third International Conference on Civil Engineering Research (ICCER)

August $1^{\text {st }}-2^{\text {nd }} 2017$, Surabaya - Indonesia

Table 11. Calculation Result of $\mathrm{NO}_{\mathrm{x}}$ Emission Rate $\left(\mathrm{qNO}_{\mathrm{x}}\right)$ Existing Condition

\begin{tabular}{lcccccc}
\hline \multirow{2}{*}{ Location } & \multicolumn{3}{c}{ Direction 1 (gr/km) } & \multicolumn{3}{c}{ Direction 2 (gr/km) } \\
\cline { 2 - 7 } & MC & LV & HV & MC & LV & HV \\
\hline $\begin{array}{l}\text { Jl. Urip Sumoharjo } \\
\text { (6/2 D) }\end{array}$ & & & & & & \\
\hline Morning & 3.0939 & 3.4561 & 3.5729 & 2.8785 & 3.3571 & 3.5263 \\
\hline Afternoon & 3.0967 & 3.4573 & 3.5735 & 2.8605 & 3.3481 & 3.5220 \\
\hline Evening & 2.9023 & 3.3687 & 3.5319 & 3.0899 & 3.4544 & 3.5722 \\
\hline Jl. Basuki Rahmat & & & & & & \\
(5/1 D) & & & & & & \\
\hline Morning & 3.3520 & 3.5577 & 3.6176 & - & - & - \\
\hline Afternoon & 3.3860 & 3.5702 & 3.6229 & - & - & - \\
\hline Evening & 3.2208 & 3.5071 & 3.5955 & - & - & - \\
\hline
\end{tabular}

Table 12. Calculation Result of $\mathrm{NO}_{\mathrm{x}}$ Emission Rate $\left(\mathrm{qNO}_{\mathrm{x}}\right)$ Plan Condition Before Tram Operates

\begin{tabular}{|c|c|c|c|c|c|c|}
\hline \multirow[t]{2}{*}{ Location } & \multicolumn{3}{|c|}{ Direction 1 (gr/km) } & \multicolumn{3}{|c|}{ Direction 2 (gr/km) } \\
\hline & MC & LV & HV & MC & LV & HV \\
\hline \multicolumn{7}{|c|}{$\begin{array}{l}\text { Jl. Urip Sumoharjo } \\
\text { (6/2 D) }\end{array}$} \\
\hline Morning & 3.1481 & 3.4790 & 3.5833 & 2.9354 & 3.3846 & 3.5395 \\
\hline Afternoon & 3.1484 & 3.4791 & 3.5834 & 2.9143 & 3.3745 & 3.5347 \\
\hline Evening & 2.9553 & 3.3939 & 3.5440 & 3.1470 & 3.4786 & 3.5831 \\
\hline \multicolumn{7}{|c|}{$\begin{array}{l}\text { Jl. Basuki Rahmat } \\
\text { (5/1 D) }\end{array}$} \\
\hline Morning & 3.3941 & 3.5731 & 3.6242 & - & - & - \\
\hline Afternoon & 3.4258 & 3.5844 & 3.6289 & - & - & - \\
\hline Evening & 3.2712 & 3.5270 & 3.6043 & - & - & - \\
\hline
\end{tabular}

Table 13. Calculation Result of $\mathrm{NO}_{\mathrm{x}}$ Emission Rate $\left(\mathrm{qNO}_{\mathrm{x}}\right)$ Plan Condition If Tram Operates

\begin{tabular}{lcccccc}
\hline \multirow{2}{*}{ Location } & \multicolumn{3}{c}{ Direction 1} & $(\mathbf{g r} / \mathbf{k m})$ & \multicolumn{3}{c}{ Direction 2 } & $(\mathrm{gr} / \mathbf{k m})$ \\
\cline { 2 - 7 } & MC & LV & HV & MC & LV & HV \\
\hline $\begin{array}{l}\text { Jl. Urip Sumoharjo } \\
\text { (6/2 D) }\end{array}$ & & & & & & \\
\hline Morning & 3.2841 & 3.5332 & 3.6072 & 3.0802 & 3.4502 & 3.5702 \\
\hline Afternoon & 3.2845 & 3.5333 & 3.6073 & 3.0588 & 3.4409 & 3.5659 \\
\hline Evening & 3.0951 & 3.4566 & 3.5732 & 3.2875 & 3.5345 & 3.6078 \\
\hline Jl. Basuki Rahmat & & & & & & \\
(5/1 D) & & & & & & \\
\hline Morning & 3.3889 & 3.5712 & 3.6234 & - & - & - \\
\hline Afternoon & 3.4208 & 3.5826 & 3.6282 & - & - & - \\
\hline Evening & 3.2655 & 3.5248 & 3.6034 & - & - & - \\
\hline
\end{tabular}

Table 14. Estimated Number of $\mathrm{NO}_{\mathrm{x}}$ Emissions Each Condition

\begin{tabular}{lcc}
\hline \multicolumn{1}{c}{ Location } & $\begin{array}{c}\text { Total } \\
\text { Average of } \\
\text { NO } \\
\text { (kg/day) }\end{array}$ & $\begin{array}{c}\text { Total Average } \\
\text { of } \mathbf{N O}_{\mathbf{x}} \text { per } \\
\mathbf{y e a r} \\
(\mathbf{k g} / \mathbf{y e a r})\end{array}$ \\
\hline $\begin{array}{l}\text { J1 Urip Sumoharjo } \\
\text { (6/2 D) }\end{array}$ & & \\
\hline Existing & 739.01 & 269739.26 \\
\hline Before Trem Operates & 887.40 & 323900.11 \\
\hline After Trem Operates & 684.45 & 249824.94 \\
\hline Jl Basuki Rahmat & & \\
(5/1 D) & & \\
\hline Existing & 1073.33 & 391766.96 \\
\hline Before Trem Operates & 1270.57 & 463759.83 \\
\hline After Trem Operates & 946.57 & 345497.30 \\
\hline
\end{tabular}


Table 15. Estimated Cost of $\mathrm{NO}_{\mathrm{x}}$ Emissions Each Condition

\begin{tabular}{lcc}
\hline \multicolumn{1}{c}{ Location } & $\begin{array}{c}\text { Cost of NO }_{\mathbf{x}} \\
\text { Emissions per } \\
\text { day } \\
\text { (Rp/day) }\end{array}$ & $\begin{array}{c}\text { Cost of NO } \\
\text { Emissions per } \\
\text { year } \\
\text { (Rp/year) }\end{array}$ \\
\hline $\begin{array}{l}\text { J1 Urip Sumoharjo } \\
\text { (6/2 D) }\end{array}$ & & \\
\hline Existing & $91,637.45$ & $33,447,668.33$ \\
\hline Before Trem Operates & $110,037.30$ & $40,163,614.15$ \\
\hline After Trem Operates & $84,872.03$ & $30,978,292.28$ \\
\hline $\begin{array}{l}\text { J1 Basuki Rahmat } \\
\text { (5/1 D) }\end{array}$ & & \\
\hline Existing & $133,093.43$ & $48,579,102.75$ \\
\hline Before Trem Operates & $157,551.29$ & $57,506,219.32$ \\
\hline After Trem Operates & $117,374.42$ & $42,841,664.92$ \\
\hline
\end{tabular}

vehicles, then analyzed the volume of vehicles during peak hour morning, afternoon, and evening. The result of existing vehicle volume analysis during peak hours can be seen in Table 2.

Based on the existing vehicle volume data from the survey results in Table 2 , it can be seen that the composition of vehicles is dominated by motorcycles and passenger car.

Furthermore, from the volume data of the acquired vehicle, then it is projected for the conditions before the tram operates (assuming operates in 2019) and when the tram operates. The result of the data projection before the tram operates are presented in Table 3 .

Percentage data transfer of modes from passenger car to tram obtained from previous research data that is equal to $25.43 \%$. Projection of vehicle volume data when tram operates can be seen in Table 4.

The next step after obtaining vehicle volume data for each condition, then performed the calculation of the conversion of data volume of vehicle / second to unit of passenger car / second by multiplying by the factor multiplier $\mathrm{NO}_{\mathrm{x}}$. The calculation result of $\mathrm{NO}_{\mathrm{x}}$ emission factor for each condition is presented in Table 5, Table 6 and Table 7.

Then after getting the $\mathrm{NO}_{\mathrm{x}}$ emission multiplier factor is to analyze vehicle speed based on road performance for each condition. Vehicle speed change is influenced by road performance. Changes in the performance of road segments are affected by changes in vehicle volume and reduced road capacity due to some road bodies used as tramway rail plans. Vehicle speed analysis results for each condition are presented in Table 8, Table 9 and Table 10.

Based on the calculation of the speed indicates that for Basuki Rahmat Street, the estimated speed of the vehicle's planned condition when the tram operates higher than the speed of the vehicle before the tram operates. As for Urip Sumoharjo Street, the vehicle speed estimates the condition of the plan when the tram operates lower than the speed of the vehicle before the tram operates.

After getting the speed of the vehicle for each condition, the next calculation of $\mathrm{NO}_{\mathrm{x}}$ emission rate. The
$\mathrm{NO}_{\mathrm{x}}$ emission rate calculations for each of the conditions are presented in Table 11, Table 12 and Table 13

Based on the calculation of $\mathrm{NO}_{\mathrm{x}}$ emissions rate, then calculated the amount of $\mathrm{NO}_{\mathrm{x}}$ emissions for each condition. The calculation is done by multiplying the $\mathrm{NO}_{\mathrm{x}}$ emission rate by the $\mathrm{NO}_{\mathrm{x}}$ emission multiplier factor.

Of the total $\mathrm{NO}_{\mathrm{x}}$ emissions of each peak hour, then averaged for the calculation of the estimated amount of $\mathrm{NO}_{\mathrm{x}}$ emissions per day. The result of calculating the amount of $\mathrm{NO}_{\mathrm{x}}$ emissions for each condition is presented in Table 14.

After knowing the amount of $\mathrm{NO}_{\mathrm{x}}$ emission for each condition, next is calculate air pollution cost due to $\mathrm{NO}_{x}$ gas emission for each condition. Based on the Minister of Environment Regulation no. 13/2011 in JAREE 2 2014: $98-111^{[3]}$, the cost of air pollution for $\mathrm{NO}_{\mathrm{x}}$ gas emissions is Rp. 124 / kg. The result of calculation of air pollution cost due to $\mathrm{NO}_{\mathrm{x}}$ gas emission can be seen in Table 15.

Based on calculations, the level of Nox reduction in the time before the tram operates when compared to when the tram operates on Urip Sumoharjo Street amounted $7.38 \%$. While the level of Nox reduction in the time before the tram operates when compared to when the tram operates on Basuki Rahmat Street amounted to $11.81 \%$

Then from the calculation of the cost air pollution, decrease the cost of air pollution due to Nox in Urip Sumoharjo Street is 9,185,321.87 rupiahs/year, while the decline in the cost of air pollution due to Nox in Basuki Rahmat Street is $14,664,554.40$ rupiahs/year.

\section{REFERENCES}

[1] Y. Kusuma, "The Influence of Fuel on Transportation Activity Against Air Pollution", Sigma-Mu Journal, Vol.5, No.1, pp. 533553, 2013.

[2] Public Works Department, "Pedoman Teknik Tata Cara Prediks Polusi Udara Skala Mikro Akibat Lalu Lintas No. 017/T/BM/1999”, Director General of Binamarga, 1999.

[3] L. R. Bestari, A. Hidayat and M. Yani, "The Estimation of Diese Vehicle's Tax Value in Relation to Air Pollution Loss (Case Study: Metro Mini in DKI Jakarta)', Journal of Agriculture Resource and Enviromental Economics, Vol.2, pp. 98-111, 2014 